\title{
A Power Controlled Interference Aware Routing Protocol for Dense Multi-Hop Wireless Networks
}

\author{
Liran Ma \\ Department of Computer Science \\ George Washington University, \\ Washington, DC 20052, USA. \\ Irma@gwu.edu
}

\author{
Qian Zhang \\ Wireless and Networking Group \\ Microsoft Research Asia, \\ P.R.China. \\ qianz@microsoft.com
}

\author{
Xiuzhen Cheng \\ Department of Computer Science \\ George Washington University, \\ Washington, DC 20052, USA. \\ cheng@gwu . edu
}

\begin{abstract}
A key issue impacting the performance of multi-hop wireless networks is the interference among neighboring nodes. In this paper, we propose a novel and practical interference aware metric, termed as Network Allocation Vector Count (NAVC), to estimate the effects of the interference on the average delay and the available bandwidth. This metric can be easily applied to routing protocols designed for 802.11 based multi-hop networks with no modification to the current 802.11 protocol. The design of NAVC as a metric for the AODV [32] routing protocol, as well as a metric for transmit power control, are described in detail. Our simulation results reveal that the NAVC-driven AODV can greatly improve its performance compared to those protocols based on hop-count. For scenarios of densely deployed nodes, the throughput improvement is often a factor near two, indicating that NAVC is more useful as networks grow larger. Moreover, the network lifetime can be notably prolonged when the NAVC is employed to conduct transmit power control. Our approach is essential for emerging applications such as wireless sensor networks where the interference is heavy and the energy is severely constrained.
\end{abstract}

Keywords: multi-hop wireless networks, interference aware routing, medium access control (MAC), power control.

\section{Introduction}

A group of autonomous wireless nodes that wish to communicate may self-organize into a multi-hop wireless network or an ad hoc network. There is neither a fixed infrastructure nor a central server in such networks. Each node acts as a router to discover and maintain routes to other nodes. Usually, nodes are powered by limited power supply, and have a fixed radio transmission range.

In multi-hop wireless networks, communications between nodes take place over radio channels. Therefore, as long as the nodes use the same frequency band for communication, any node-to-node transmission adds to the level of interference experienced by other nodes in vicinity. As a result, network capacity, connectivity, link quality, and bandwidth availability, vary dynamically on a variety of timescales in different parts of the network. These problems are compounded further when communicating nodes are not within direct transmission range of each other, and when the network size grows.

It is shown by Gupta and Kumar [19] that sharing the same radio channel among the neighboring nodes is the fundamental reason that leads to the degradation of performance when the number of nodes increases. Therefore, finding a practical wireless interference aware metric to improve the system performance is critical since contention from neighboring nodes for the shared channel is severe when nodes are densely deployed. The interference aware metric can be employed by the network layer to make routing decisions, the Medium Access Control (MAC) layer to schedule transmissions, and the physical layer to conduct power control.

Many of the recent routing protocols (e.g. [24, 25] and [32]) choose routes with minimum hop-count, ignoring the possibility that a longer path might offer higher throughput. Further, the arbitrary selection of 
one path from all those with minimum hop-count disregards the often large differences among these paths. As indicated by Das et al. [9], hop-count based protocols only achieve a small portion of the network capacity. For example, in one of their simulated network scenarios containing 100 nodes with $2 \mathrm{Mbps}$ links, the throughput of each node is on the order of a few kilobits per second [9]. The pure hop-count metric is misleading in long term because it may choose a route with significantly less capacity than the best one in the network [11]. This is particularly true for dense networks when multiple paths with the same hop-count exist.

Lately, a metric for high-throughput path computation is developed in [10]. This metric is termed as Expected Transmission Count (ETX), which is obtained from link loss characteristics between the two directions of each link and the interference among the successive links of a path. An ETX-driven routing protocol can substantially improve the system performance. Nevertheless, in scenarios involving mobile senders, minimum hop-count routing performs considerably better because ETX does not react sufficiently quickly [14]. A power aware metric is introduced in [34]. This metric takes into consideration the energy consumed per-packet, the time to network partition, and the variance in the battery life of all nodes. PARO, proposed in [17], constructs a power-efficient route via intermediate "redirectors" to reduce the aggregated transmit power. Other power-aware routing schemes have also been studied in [3], [26] and [31].

However, none of the above-mentioned works employ the interference situations as the core metric when building paths. As it is claimed in [23], there is an opportunity for achieving throughput gains by exploiting an interference aware metric. The impact of interference on the performance and the capacity of multihop wireless networks has been extensively studied in literature ([12, 13, 23] and [20]). Nevertheless, all these state-of-the-art works limit their research in theoretical analysis. To our best knowledge, no proper interference aware metric has been derived. There exist significant challenges in finding an interference aware metric for the network layer to improve the overall performance.

In addition to those efforts in developing more efficient routing protocols, a distributed channel-access technique based on a new model to analyze the interference in large-scale dense packet radio networks has been proposed in [33] by Shepard. In this study, the author considers the limit on the capacity imposed by the aggregate interference from many senders spreading over a large area, and concludes that the capacity can be increased with minimum-energy routing. Our work, in contrast, focuses on a novel interference aware metric that can be applied to the existing 802.11-based channel access algorithm, which can not easily support the minimum-energy routing.

To our best knowledge, this paper is the first to propose a pragmatical interference aware metric that is not only for routing in the network layer, but also for controlling transmit power in the physical layer. Our proposed metric can be easily applied to routing protocols designed for 802.11 based multi-hop networks with no requirement of modifying the current 802.11 protocols. The main contributions of our work are multifold:

1. We propose a novel and practical interference aware metric termed Network Allocation Vector Count (NAVC) for multi-hop wireless networks.

2. We design the NAVC-driven AODV protocol, the marriage of NAVC as a routing metric and the classical AODV protocol [32].

3. We develop a fully distributed transmit power control policy, which functions as a part of the NAVCdriven AODV protocol, based on NAVC and the number of neighboring nodes.

4. We show that in scenarios of densely deployed networks with moderately high traffic load (more than $70 \%$ of the maximum capacity is utilized for data transmission), our NAVC-driven AODV outperforms traditional AODV though the former uses longer paths.

5. Finally, we demonstrate that using NAVC can substantially increase the network lifetime by saving the transmit power in cases of densely deployed networks. 
The remainder of this paper is organized as follows. In Section 2, we develop a system model for the interference analysis, from which our NAVC metric is derived. The NAVC-driven AODV routing scheme is discussed in detail at Section 3. Section 4 reports our simulation study and Section 5 concludes our works.

\section{Interference Analysis and Metric}

In this paper, we adopt the point-to-point coding model of Gupta and Kumar [19] to analyze the interference among neighboring nodes. In this model, a receiver only decodes the message from one sender at any given time, considering other simultaneous transmissions purely as noise. Similarly, at any given time, a sender transmits information only to one receiver. To be more specific, each node interferes with its neighborhood when sending packets, while encounters interference from its neighbors when they are transmitting.

Variations in network size (the number of nodes), network density (relative positions of nodes), and traffic per node could have strong influence on the interference experienced by nodes throughout the network. We focus on the aggregate interference imposed by both self-generated traffic at each node, and relay traffic that is hopping throughout the network. The amount of the relay traffic in the network depends directly on the number of hops from an arbitrary source to any other destination.

Subsequently, we choose to characterize the interference via studying the traffic in vicinity using fluid model. To our best knowledge, fluid model was first introduced to analyze data-handling systems with multiple sources in [2]. In this model, discrete packets within bursts are modeled as a continuous fluid. The continuous nature of fluid makes this model more analytically tractable. In wireless multi-hop networks, each node operates not only as an end-system, but also as a router to forward packets. Therefore, this type of networks can be considered as a special case of data-handling systems such as the Internet. Thus the fluid model is applicable in this study.

In [7], the Poisson Counter Driven Stochastic Differential Equation (PCDSDE) has been introduced to analyze fluid queueing systems. Next, we will use the PCDSDE to demonstrate how the surrounding traffic influences the queueing behaviors of an arbitrary node in a wireless multi-hop network.

\subsection{Definition of PCDSDE}

To define PCDSDE, consider the following stochastic integral equation:

$$
x(t)=x(0)+\int_{0}^{t} f(x(\tau), \tau) d \tau+\int_{0}^{t} g(x(\tau), \tau) d N_{\tau},
$$

where $N_{\tau}$ is a Poisson Counter. The following definition is given in [7].

Definition: $x(\cdot)$ is a solution of (1) in the Itô sense if, on an interval where $N$ is a constant, $x$ satisfies $\dot{x}=f(x, t)$, and if, when $N$ jumps at $t_{1}, x$ changes according to

$$
\lim _{t \rightarrow t_{1}^{+}} x(t)-\lim _{t \rightarrow t_{1}^{-}} x(t)=g\left(\lim _{t \rightarrow t_{1}^{-}} x(t), t_{1}\right)
$$

where $x(\cdot)$ is assumed to be continuous from the left. From Eq.(1), we obtain

$$
d x(t)=f(x, t) d t+g(x, t) d N
$$

Eq. (3) is called the PCDSDE.

Note that a stochastic differential equation may be driven by multiple Poisson counters:

$$
d x(t)=f(x, t) d t+\sum_{i=1}^{m} g_{i}(x, t) d N_{i} .
$$




\subsection{Interference Analysis}

Suppose each source node is surrounded by a number of independent neighboring ON/OFF sources. The reason for choosing $\mathrm{ON}-\mathrm{OFF}$ flow model is that in communication networks many real time applications exhibit ON-OFF characteristics. Assume the sending rate of the source is regulated by an ON-OFF process $x(t)$. According to Eq. (4), we get

$$
d x(t)=(1-x(t)) d N_{1}(t)-x(t) d N_{2}(t), \quad x(0) \in(0,1) .
$$

Eq. (5) does not have the $f(x, t)$ term compared to Eq. (4) but involves two Poisson Counters $N_{1}$ and $N_{2}$ representing the $\mathrm{ON}$ and the OFF status, respectively. It is observed that $x(t)$ remains unchanged on an interval where $N_{1}$ and $N_{2}$ are constants. When $N_{1}$ or $N_{2}$ does jump, $x(t)$ flips between 0 and 1 .

Assuming $\lambda$ and $\mu$ are the rates of $N_{1}$ and $N_{2}$ respectively, we can easily obtain the expectation of $x(t)$ in the steady state:

$$
E[x(t)]=\frac{\lambda}{\lambda+\mu} .
$$

Note that $x(t)=1$ means that the radio channel is occupied by the neighborhood traffic, and thus the source node inserts packets (fluid) into its buffer at a rate $h$. When $x(t)=0$, the radio channel is reserved by this source node and the packets queued in the buffer will be sent out. For simplicity, we assume that each node sends out buffered packets at a constant rate $c$, which could be smaller than $h$. The queue length $v(t)$ at a source node can be described by Eq. (7):

$$
d v(t)=-c I_{v} d t+h x(t) d t
$$

where $h x(t)$ indicates the influence of the interference from neighboring nodes on the rate at which packets are inserted into the queue. The notation $I_{v}$ in Eq. (7) is the indicator function for $v>0$, while $v$ is the queue length. Subsequently, we may consider the following expectation:

$$
d E[v(t)]=-c E\left[I_{v}\right] d t+E[h x(t)] d t .
$$

Dividing both sides of (8) by $d t$ leads to

$$
\frac{d}{d t} E[v(t)]=-c E\left[I_{v}\right]+E[h x(t)]
$$

From Eq. (9), we get

$$
E[v(t)]=\left(c-h \frac{\lambda}{\lambda+\mu}\right)^{-1} \frac{h-c}{\lambda+\mu} E[h x(t)] .
$$

According to the Itô formula, the Chain Rule, and Eqs. (6) and (10), we obtain Eqs. (11) and (12):

$$
\begin{gathered}
\frac{d}{d t} E\left[v^{2}(t)\right]=-2 c E[v(t)]+E[h x(t)] . \\
E\left[v^{2}(t)\right]=2 c\left(c-h \frac{\lambda}{\lambda+\mu}\right)^{-1} \frac{h-c}{\lambda+\mu} E[v(t)] .
\end{gathered}
$$

Note that $h \lambda /(\lambda+\mu)<c$, which means that the average packet generation rate ought to be lower than the average packet sending rate. Otherwise, the whole system can not work properly. From Eqs. (10) and (12), an important conclusion can be drawn: when the value of $1 /(\lambda+\mu)$ keeps constant, the node's queue length increases with $\lambda /(\lambda+\mu)$. This implies that the burstness characteristics of the traffic originating from the neighboring nodes is the major factor impacting the source node's queueing delay given that $1 /(\lambda+\mu)$ is a constant.

Naturally, we need to find a measurable parameter that characterizes the $\lambda /(\lambda+\mu)$, i.e., the interference level caused by neighboring traffic. Since it is not practical to gauge the radio strength directly from the physical layer, identifying an alternative solution is necessary. Next, we will describe how to derive our interference aware metric from the MAC layer. 


\subsection{Interference Aware Metric}

It is well known that the principal MAC technique of IEEE 802.11 [21] is called Distributed Coordination Function $(D C F)$. DCF exploits a random back-off procedure through physical carrier sense to resolve medium contention conflicts and virtual carrier sense for exchanging request-to-send (RTS) / clear-to-send (CTS) frames to announce the impending use of the medium. Naturally, information contained in the RTS/CTS handshake protocol can be used to measure the traffic in vicinity.

The Markov Chain model, a well-known analytical model for the binary slotted exponential back-off process in IEEE 802.11 DCF [5], will be applied. Without loss of generality, we assume each node has $n$ neighbors. Let $\tau_{i}$ be the probability that node $i$ transmits in a randomly chosen time slot, and $p_{i}$ be the collision probability observed by a packet being transmitted from node $i$. In other words, $p_{i}$ is the probability that a packet encounters interference. According to [5], $p_{i}$ is assumed to be constant and independent for every transmission attempt, regardless of the number of retransmissions suffered. By extending the results in [5], we obtain

$$
\tau_{i}=\frac{2}{1+W+p_{i} W \sum_{j=0}^{m-1}\left(2 p_{i}\right)^{j}},
$$

where the Contention Window $(\mathrm{CW})$ size $W$ varies form $C W \min$ to $C W \max \left(C W \max =2^{m} C W \min \right)$. According to Eq. (13), $\tau_{i}$ depends on the collision probability $p_{i}$, which can be expressed as:

$$
p_{i}=1-\prod_{j=1}^{n}\left(1-\tau_{j}\right) .
$$

For simplification, we might as well assume that all the nodes have the same outgoing traffic pattern. When the system is in a steady state, we have $\tau_{i}=\tau$ and $p_{i}=p_{\text {occupied }}$ because each transmission "sees" the system at the same state. Thus, the probability $p_{\text {occupied }}$ can be considered as the possibility that a transmitted packet encounters interference, i.e., the probability that at least one of the $n$ neighboring nodes transmits in a given time slot:

$$
p_{\text {occupied }}=1-(1-\tau)^{n} \text {. }
$$

Obviously, $p_{\text {occupied }}$ is a function of $\frac{\lambda}{\lambda+\mu}$, the expectation of the medium occupancy derived in Section 2.2 . More importantly, $p_{\text {occupied }}$ can be effectively estimated by employing the Network Allocation Vector (NAV) information of the 802.11 MAC layer. In our study, we use NAV Count (NAVC), defined by Eq. (16), to approximate $p_{\text {occupied }}$ :

$$
\mathrm{NAVC}=\frac{\sum_{t_{i}=t_{\mu}}^{t_{i}=t_{\nu}} \mathrm{NAV}_{t_{i}}}{t_{\nu}-t_{\mu}}
$$

where $t_{\nu}$ and $t_{\mu}$ are the bounds of the time window.

Note that once a node hears a transmission from another node in 802.11 , its NAV will be set to a busy state and this node has to keep silence for a duration equal to the value in the Duration-ID field of the header in the received RTS/CTS. Within a given observation time window, the higher the traffic rate, the larger the accumulated NAV duration, and vice versa.

With comprehensive simulation study and analysis [36], we have found that there is a fixed relationship between NAVC, as computed by Eq. (16), and the average delay and available bandwidth, as computed by Eqs. (17) and (18):

$$
\begin{gathered}
\operatorname{Delay}(x)=\left\{\begin{array}{cl}
2 \mathrm{~ms}, & 0 \leq x \leq 0.2 \\
2 e^{7.9(x-0.2)^{2} \mathrm{~ms},} & 0.2<x \leq 0.65
\end{array}\right. \\
B W(L, x)=(0.0024 L+0.9)-(0.0036+1.4) x \quad \mathrm{Mbps},
\end{gathered}
$$

where $L$ is the packet length and $x$ represents the NAVC value. This relationship is insensitive to the number of users and the traffic pattern. That is, once we observe a NAV value during a time window, the available bandwidth and access delay can be estimated given a certain packet length. For the detailed derivation of Eqs. (17) and (18), we refer the readers to our previous papers [28] and [36]. 
If NAVC is less than 0.2 , the average delay is usually ignorable. When NAVC is greater than 0.65 , it indicates that the node has been overloaded. Thus, as long as the NAV value can be sensed from the MAC layer, we are able to estimate the delay characteristics by Eq. (17). In addition, if the average packet length is available, the available bandwidth $(\mathrm{BW})$ can be approximately estimated via Eq. (18). NAVC vs. average delay and NAVC vs. available bandwidth are plotted in Figs. 1(a) and 1(b) respectively, based on Eqs. (17) and (18).

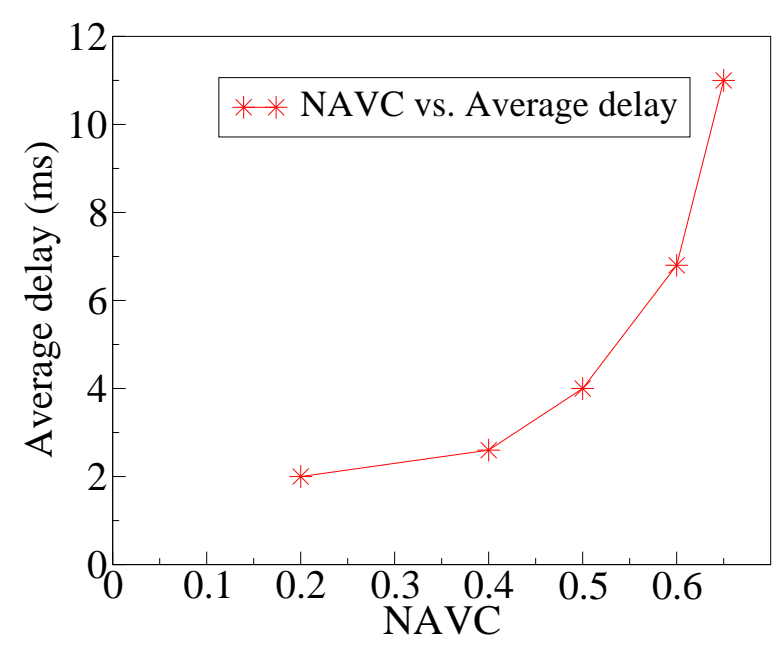

(a) NAVC vs. Average delay

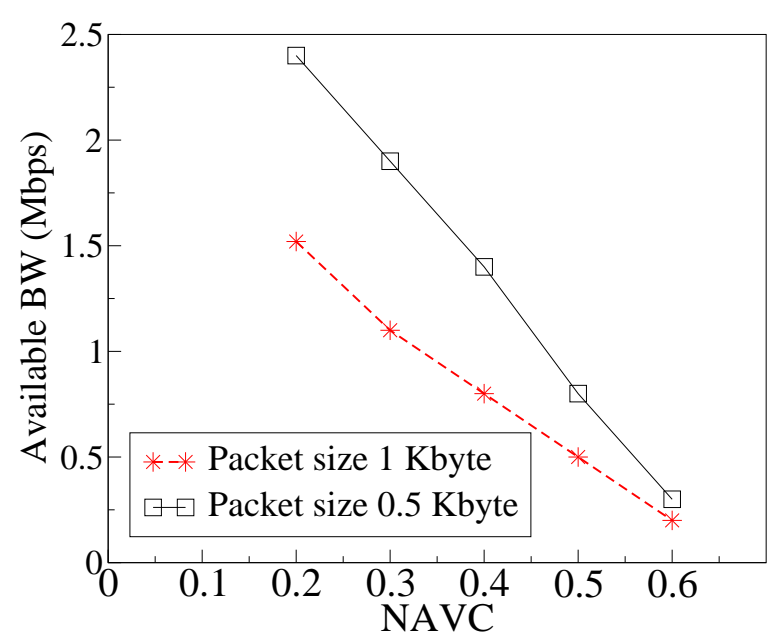

(b) NAVC vs. Available bandwidth

Figure 1: NAVC vs. Delay and Available Bandwidth.

\section{NAVC-Driven AODV}

Now we have introduced the system model and the new interference aware metric NAVC. Before we elaborate the new NAVC-driven AODV protocol that selects a path based on the NAVC metric, we will first give a brief overview on the core of AODV [32].

\subsection{Overview of AODV}

AODV is a reactive routing protocol proposed for mobile ad hoc wireless networks. It computes the route ondemand with a tradeoff of extending the delay time of the first data packet. Compared to proactive routing protocols, where routes to all possible destinations are stored within each node and updated when topology changes, reactive routing protocols have less protocol overhead and better scalability. As an interference aware metric, NAVC can be applied to both reactive and proactive routing protocols. In this paper, we choose to study the NAVC-driven AODV protocol as an example, mainly due to the fact that AODV is well-studied by the ad hoc wireless network society.

In AODV when a source node desires a route to a destination for which it does not have a route, it uses a route discovery process to dynamically determine such a route. The route discovery is based on a query and reply cycle and the route information is stored in all intermediate nodes on the route in the form of route table entries. Route discovery is performed by flooding the network with ROUTE REQUEST (RREQ) packets.

Each intermediate node continues broadcasting the $R R E Q$ unless this node is the destination or it has a route entry to the destination in its route table. Such a node responses to the $R R E Q$ by generating a 
ROUTE REPLY (RREP) packet that is routed back to the original source. The RREQ builds up the path traversed so far and the $R R E P$ routes itself back to the source along a reversed path.

Once the source node receives the $R R E P$, it can start the data packet transmission. If the source later receives a $R R E P$ with a better metric, that is, a greater destination sequence number, or the same sequence number but with a smaller hop-count, it may update its routing information for that destination and begin using the better route.

AODV maintains a route as long as the source has data to the destination along that path. Once the source stops sending data packets, the route will timeout and eventually be deleted from the routing tables of intermediate nodes.

If a link break occurs, the node upstream of the break propagates a ROUTE ERROR ( $R E R R)$ message to all nodes using that link, informing of the now unreachable destination(s). After receiving an $R E R R$, a node removes all the routes using that link. If the source node still desires the route, it can initiate the route discovery procedure again.

It has been shown via simulation that AODV works well on small to medium networks [6]. In the next section, we will describe our routing scheme that selects a path based on the NAVC metric.

\subsection{The NAVC-Driven AODV Protocol}

As an extension to AODV, the NAVC-driven AODV is also an on-demand algorithm, constructing routes using a route request and route reply cycle with the interference aware metric NAVC. The key idea is to avoid selecting a route that has nodes residing in a heavily interfering neighborhood so that the energy and time spent in contention is minimized. Further, the severely interfered nodes in a path will starve downstream nodes in the chain.

Each node periodically computes a NAVC value by collecting the NAV value from its MAC layer. With this metric, the route selection and power control decision can be made at the network layer with the interference information obtained from the link layer. Route selection based on NAVC alleviates the reduction of the throughput due to the interference along the whole path.

Specifically, the modified routing protocol contains two operating modules: route discovery and transmit power control.

\subsubsection{Route Discovery}

The route discovery procedure includes three components: route request, route reply, and route maintenance.

\section{Route Request}

There are two additional options namely heavy_node_number and nav_sum in the $R R E Q$ message in addition to all the options in the normal AODV RREQ message. After receiving the $R R E Q$ packet, each node other than the destination has three possible activities:

- If its NAVC is larger than 0.65 , increase the option heavy_node_number by 1 because this node is considered as a heavily interfered node ${ }^{1}$. In addition, increase the option nav_sum by the square of the NAVC value. The purpose of using square value is to differentiate the NAVC value by adding increasing weight.

- If its NAVC is between 0.25 and 0.65 , increase only the option nav_sum by the square of the NAVC. Whenever a node's NAVC is less than 0.25 , it does not make significant difference in terms of average delay and residual bandwidth for a link ${ }^{2}$.

- If none of the above is satisfied, continue broadcasting this $R R E Q$ without making any modification to the two options.

\footnotetext{
${ }^{1}$ Refer to Section 2.3 .

${ }^{2}$ Refer to Section 2.3.
} 


\section{Route Reply}

When there are multiple $R R E P$ s for the same communicating pair, the path with the least heavy_node_number and nav_sum value will be selected. The heavy_node_number gets higher priority compared to the nav_sum in making path selection.

\section{Route Maintenance}

When any of the following conditions holds, the route update process will be activated at the source, the destination or an intermediate node.

- Receives another valid RREP notifying a better route. Here a "better route" means a route with lower heavy_node_number; or lower nav_sum with the same heavy_node_number; or less hop count with the same heavy_node_number and nav_sum.

- Receives a ROUTE RENEW message

- Receives a ROUTE ERROR message.

- Route entry times out.

Otherwise, retain the most recently used paths for forwarding packets.

\subsubsection{Transmit Power Control}

The primary goal of the transmit power control is to reduce the radio interference and increase the spatial reusability, which usually generates better paths in the network. On the other hand, transmission to a distant device at a higher power level consumes a disproportionate amount of power compared with the transmission to a node in closer proximity [17]. Thus transmit power control can save energy.

However, the reduction of the transmit power may introduce unidirectional links, which may affect the connectivity to a great extent and degrades 802.11 MAC performance [29]. Therefore, the transmit power must be adjusted such that a node not only can receive a packet addressed to it, but also can successfully participate in the higher-level routing protocol. Subsequently, the underlying routing protocol should be capable of rapidly responding to these changes using minimum signaling.

Transmit power control consists of two phases, namely the neighbor discovery and transmit power adjustment.

\section{Neighbor Discovery}

Neighborhood information can be obtained by exploiting the HELLO messages. Each node periodically broadcasts a HELLO message, which contains the node's NAVC value and its current transmit power level (denoted by $\mathrm{Cur}_{t r}$ ). Upon receiving a $H E L L O$ message, the node computes the minimum transmit power level (denoted by $M i n_{t r}$ ) necessary to reach the source of the $H E L L O$ message based on the received signal strength. If its $\mathrm{Cur}_{t r}$ is larger than the computed $\mathrm{Min}_{t r}$, the source node is qualified as a neighbor. Otherwise, the source node is not considered as a neighbor because this link is unidirectional. With this simple protocol, a node can obtain important information including the number of neighboring nodes covered by its $C u r_{t r}$ and their interference situation.

\section{Transmit Power Adjustment}

Each node conducts power control based on its NAVC value and the number of neighbors. We assume that each wireless LAN card has multiple transmit power levels so that the output power can be adjusted in a discrete fashion.

After receiving HELLO messages from all neighboring nodes, a node starts the transmit power adjustment procedure, which is to determine whether to reduce or raise its $C u r_{t r}$. The decision is made according to the following power level adjustment eligibility: 
1. If its $\mathrm{Cur}_{t r}$ can cover eight or more neighboring nodes, which means that there are more than enough neighboring nodes to guarantee connectivity $([4,16]$ and $[35])$, the node should reduce its transmit power to the next available level. Consequently, the node should update its neighbor list and route entries constructed under the previous transmit power level. All the nodes in the neighbor list will be reexamined based on the new transmit power level and those unqualified nodes will be removed from the list. Furthermore, all the current valid route entries associating with the removed nodes will be considered as invalid so that unidirectional link is avoided.

2. If its $\mathrm{Cur}_{t r}$ can cover five to seven neighboring nodes, and half or more of them have their NAVC values exceed 0.65 , which indicates that the neighborhood is experiencing a serious interference, the node should reduce its transmit power to the next available level. Correspondingly, the node should update its neighbor list and route entries constructed under the previous transmit power level.

3. If its $\mathrm{Cur}_{t r}$ can only cover four or less number of neighbors, this node needs to increase its transmit power to the next available level in order to maintain the connectivity. Similarly, its neighbor list and route entries must be updated accordingly. All the existing valid route entries will be still considered as valid because raising transmit power will not cause unidirectional links to them. Besides, the newly qualified nodes will be added into the neighbor list.

4. If the NAVC value of a node is low, which possibly means that this node continues obtaining the radio channel, there is a danger that its neighboring nodes are captured [27]. On the contrary, if a node continually fails in reserving the channel, its NAVC value could be extremely large. In this case, the node should raise its $\mathrm{Cur}_{t r}$ in order to break the possible capture effects [22].

5. Every node should wait for some time to start the next transmit power adjustment after the current one is finished. The goal of this delay for power adjustment is to mitigate the problem of the potential frequent oscillations.

In the coming section, we will demonstrate how our algorithm achieves the desired objective of improving the total throughput in a fully distributed manner through simulation.

\section{Simulation Study}

In this section, we describe the simulation set-up and report our simulation results. By extending the AODV routing protocol, we obtain the NAVC-driven AODV via the implementation of the interference aware NAVC metric. We then study the performance improvement of the NAVC metric by comparing the performance of the pure AODV routing protocol with that of the NAVC-driven AODV.

\subsection{Simulation Settings}

Our simulation is based on the Network Simulator (ns-2) designed at the Lawrence Berkeley National Laboratory [1] with its wireless extension developed by the Monarch Project [18]. This simulator contains the source code for the core of the AODV routing protocol. To be consistent with previous works $[6,8,9,10,17]$, we have adopted the following parameters and models in our simulation.

\subsubsection{Traffic and Mobility model}

- The mobility model is the random waypoint model [6]. Nodes are placed according to a Poisson point process over a planar field with length and width of $1000 \mathrm{~m} \times 450 \mathrm{~m}$.

- Each node moves from a random location to a random destination at a randomly chosen speed, which is uniformly distributed between 0 and 10 meters/sec. 
- Traffic sources are Constant Bit Rate (CBR). The ns UDP(CBR) source and sink agents are attached to the communication pairs, which are randomly chosen from the entire network.

- The number of source-destination pairs and the packet sending rate for each pair are changed to achieve a variable offered load in the network. Each pair of UDP sessions start at staggered times.

- We fix the data packet size to be 512 byte.

\subsubsection{Wireless Model}

- Signal propagation is based on a two-ray ground model, which is appropriate for outdoor environments where a strong line of sight signal exists between the transmitter and the receiver and where the antennas are omnidirectional.

- We adopt the point-to-point coding model and the physical model of [19]. The physical model is based on the signal to interference ratio, which indicates that a packet is considered as successfully received if the received signal strength is above a certain threshold.

- Radio link bandwidth is set to $2 \mathrm{Mbps}$ with a nominal range equal to 250 meters.

\subsubsection{Energy Model}

- We assume radios are capable of dynamically adjusting their transmit powers.

- The energies consumed at different modes are not the same. According to [8] and [15], we set the energy consumption ratios of idle:receive:transimit to be 1:1.25:1.875.

- The Aironet PC4800 wireless LAN card has five discrete Radio Frequency (RF) output power levels (RF_level) [17]. We assume nodes in our simulation are equipped with this type of wireless LAN card. Therefore, each node has five $R F_{-}$levels, namely 1.3, 7.2, 37, 115, and 282 milliwatts such that its radio can reach $50,100,150,200,250$ meters respectively ${ }^{3}$.

- The transmit power $(T x)$ is computed via Eq. ${ }^{4}(19)$. The corresponding values are listed in Table 1.

$$
T x=1200+\text { RF_level. }
$$

- At the beginning of every single simulation, each node has an initial energy of 300 Joules and uses the maximum power level. We assume that the switch between any two power levels takes no time.

\begin{tabular}{|c|c|c|c|c|}
\hline Tx level 1 & Tx level 2 & Tx level 3 & Tx level 4 & Tx level 5 \\
\hline $1201 \mathrm{~mW}$ & $1207 \mathrm{mV}$ & $1237 \mathrm{~mW}$ & $1315 \mathrm{~mW}$ & $1482 \mathrm{~mW}$ \\
\hline
\end{tabular}

Table 1: Tx (Transmit) power values of 2Mbps.

We report our simulation results in the next subsection. For better elaboration, we use NAVC to refer to the NAVC-driven AODV whenever the ambiguity can be resolved through the context.

\subsection{Results}

This subsection presents the comparison results, the average of 200 runs, between the NAVC and the classical AODV in terms of the throughput, the network lifetime, and the average hop-count. The performance of a routing protocol can be measured primarily by the achievable throughput given a set of constrained resources. Other metrics such as the network lifetime and the average hop-count are also important in providing insight for better understanding of the behavior of the protocol.

\footnotetext{
${ }^{3}$ These values are computed via the two-ray ground model in $n s-2$.

${ }^{4}$ The value 1200 sums the energy consumed by different components of WLAN card [30].
} 


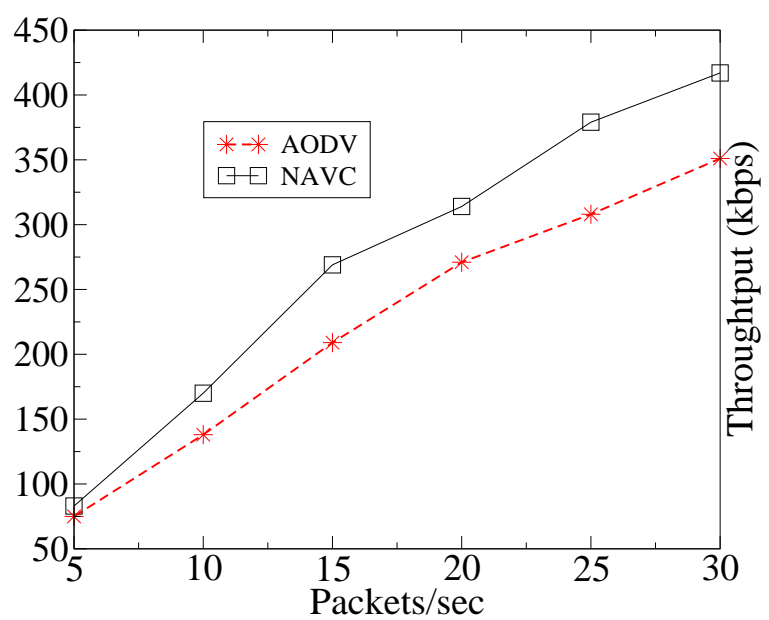

(a) 5 pairs, varying load

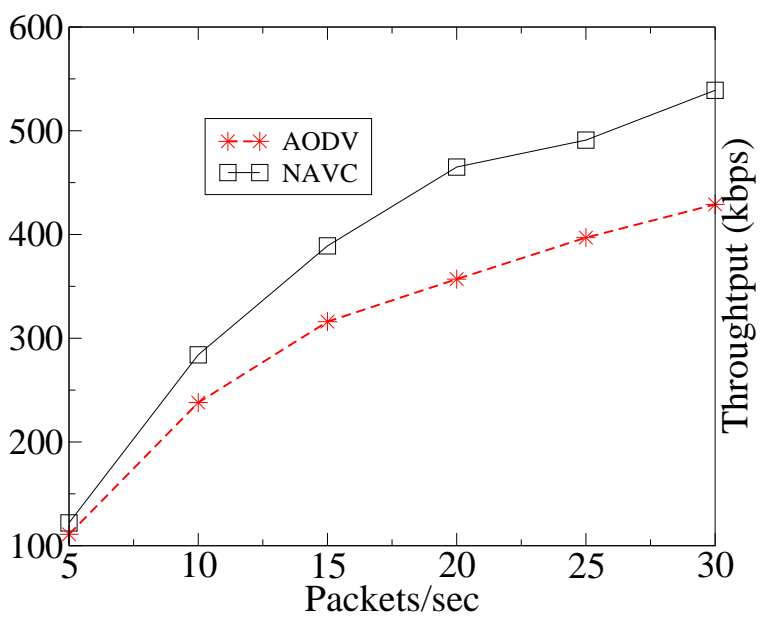

(c) 8 pairs, varying load

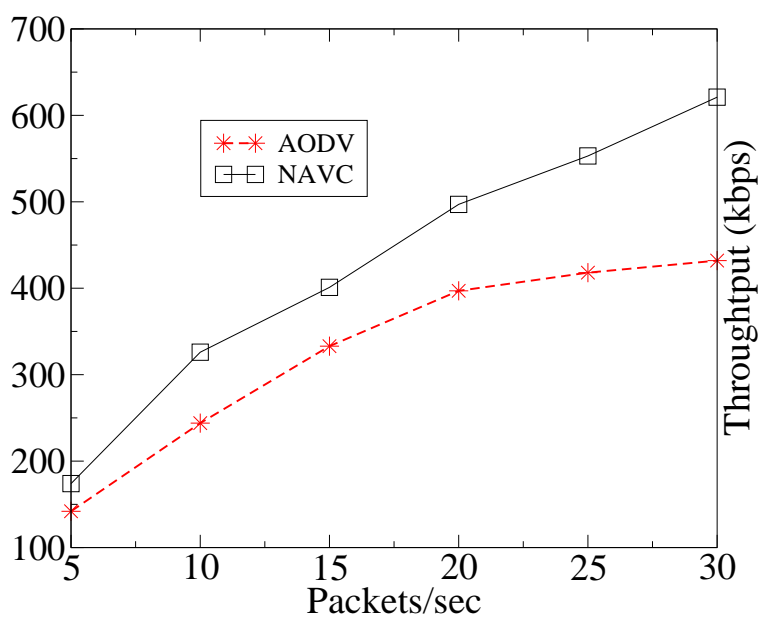

(e) 10 pairs, varying load

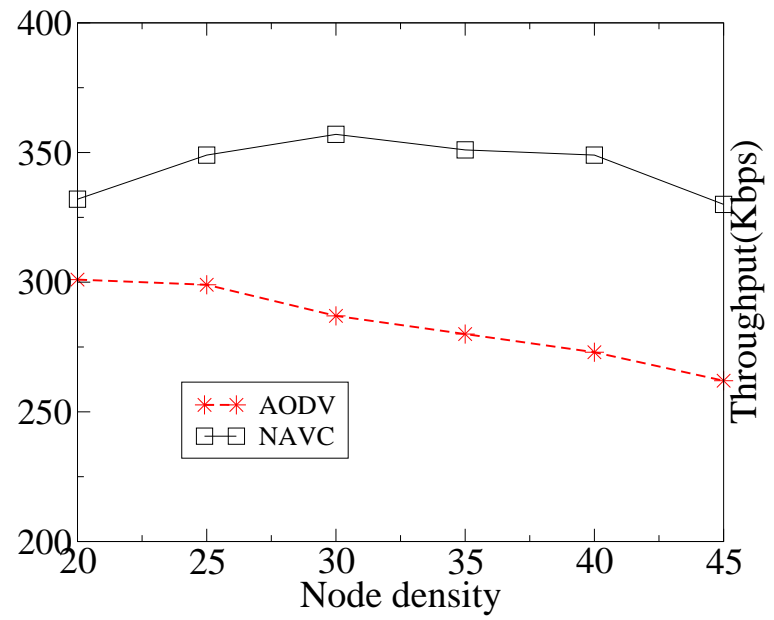

(b) 10 pairs, varying node density

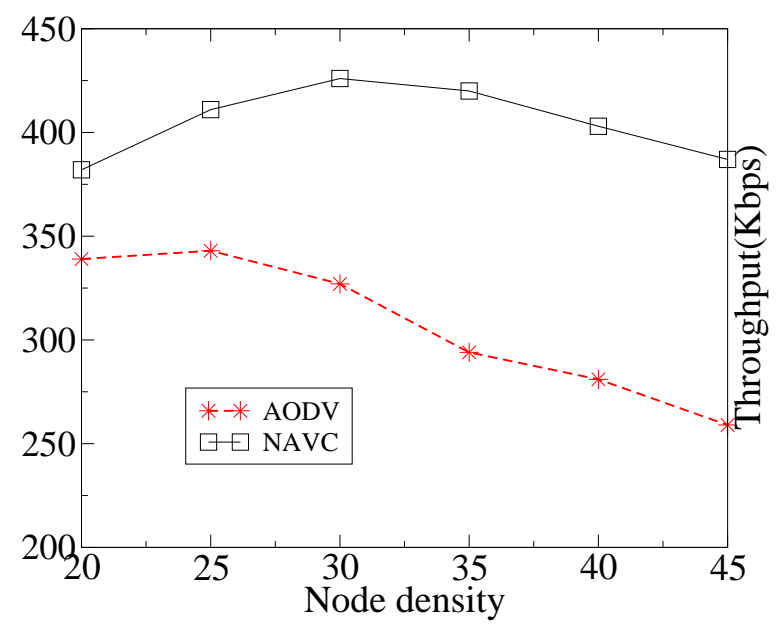

(d) 15 pairs, varying node density

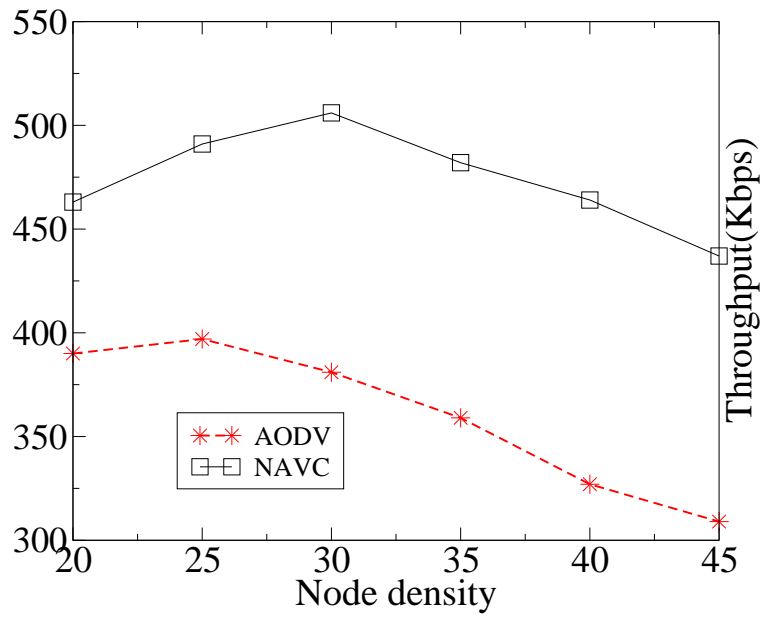

(f) 20 pairs, varying node density

Figure 2: The throughput comparison of NAVC and AODV. 


\subsubsection{Throughput}

Here, throughput refers to all the data bytes received per second at destination nodes without duplication. We evaluate the NAVC's ability to improve the throughput under different traffic load and different degree of node density. The node density [8] is defined as the average number of nodes within the radio range (i.e., an area of $250^{2} \times \pi$ square meters) other than the source and the destination node.

The first set of results is obtained when the network contains 5,8 , or 10 communication pairs with different loads. The packet rate is selected from $5,10,15,20,25$, to 30 packets/sec because we would like to offer a reasonably high load to the network. By default, the pause time in the waypoint mobility model is 60 seconds. The detailed results are plotted in Fig. 2(a), Fig. 2(c), and Fig. 2(e).

The second set of results is obtained when node density varies from $20,25,30,35,40$, to 45 . There are 10,15 , and 20 communication pairs with a fixed rate of 10 packets/sec. The detailed results are plotted in Fig. 2(b), Fig. 2(d), and Fig. 2(f).

These results demonstrate the following facts:

- NAVC exhibits higher throughput under heavier load in general.

- The denser the network is, the higher the throughput improvement NAVC can achieve. For the best case, the improvement of the total throughput is around a factor of two.

- The throughput improvement is the best at moderately heavy network load (more than $70 \%$ of the maximum capacity is utilized for data transmission) and negligible at very low load (lower than 30\% of the maximum capacity is utilized for data transmission).

- When the node density is neither too low nor too high, the NAVC works the best.

- The average throughput increases by up to $29 \%$ for UDP CBR traffic.

Yet we also observe that in scenarios involving high mobility, the proposed scheme does not react to the network changes sufficiently quick.

\subsubsection{Network Lifetime}

This subsection exams the NAVC's ability to save energy by reducing the transmit power and distributing traffic load. If the traffic load can be balanced over the entire network, the battery power of some critical nodes that would otherwise be involved in more paths will be conserved. The network lifetime is defined to be the time for the first node to drain out its energy. The larger this value is, the more energy-efficient the routing protocol is, and vice versa.

The results, shown in Fig. 3(a), are obtained from one single set of experiments with 15 communication pairs at node densities of $20,25,30,35,40$, and 45 . The source nodes start with 2000 Joules so that the traffic load can last for enough time.

Fig. 3(a) indicates that the network lifetime can be greatly improved compared to the pure AODV protocol because the NAVC can help to distribute the traffic more fairly in the whole network. The average increase in the network lifetime is about $27 \%$, which could be further improved if we also consider power saving modes such as the sleeping mode in our simulation. Typically, more power is consumed in transmitting compared with receiving and idling. As it is shown by [15], the Idle mode occupies a significant amount of the total energy because the radio electronics must be powered on to decode the received signal in order to detect the presence of an incoming packet.

Therefore, it is desirable to completely shut down the radio rather than switching to a different transmission range. However, the non-negligible transition time (e.g. 7 milliseconds for Aironet PC4800) [17] must also be taken into consideration. Under certain circumstances, frequently turning the radio on and off would result in even more energy consumption than leaving the transceiver unit in the Idle mode.

However, the main goal of reducing transmit powers here is to increase the spatial channel reusability because the sensing range is much larger than the transmission range [17]. In order to correctly receive a 


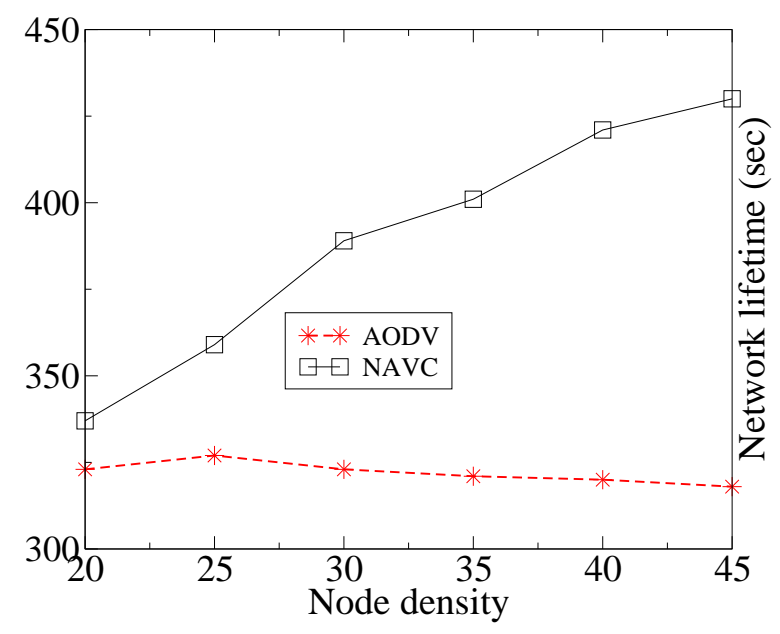

(a) Network lifetime.

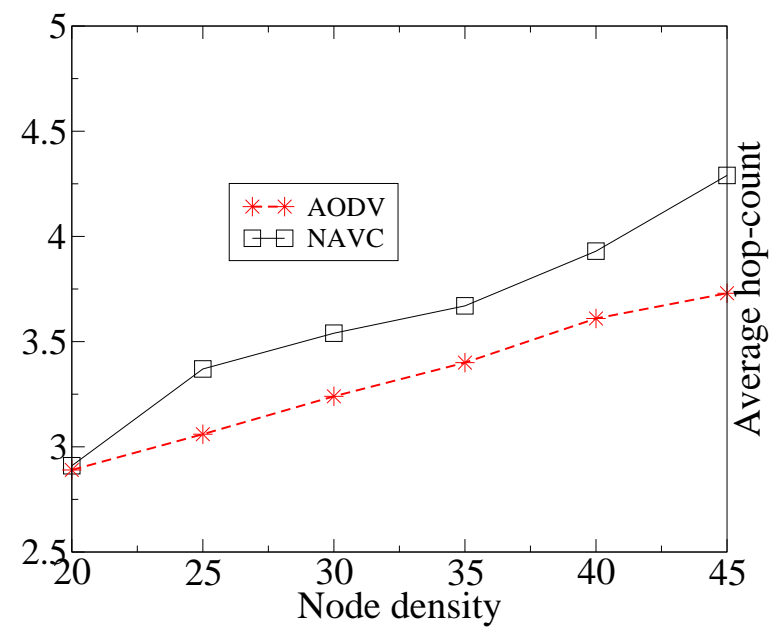

(b) Average hop-count.

Figure 3: Network lifetime and Average hop-count comparisons.

packet, the level of signal to interference ratio (SIR) should not be beyond the threshold a node can tolerate. Therefore, it is desirable to have the sensing range closer to the receiving range in order to increase the spatial reuse in the network. It is very important to observe that power control is an essential part of an interference aware routing. Under the condition with no transmit power control, the average throughput improvement is only $15 \%$.

\subsubsection{Average Hop-Count}

It is shown in Fig. 3(b) that NAVC often finds paths with larger hop-count compared to AODV. This is particularly true when the source and the destination node are farther away from each other. When the network load is light or the network is sparse, NAVC selects paths with almost the same length as AODV does. This is because either the traffic is not heavy enough to cause heavy interference or there is no forwarding nodes to form better paths. As the traffic load and node density increase, NAVC starts to choose better paths with less interference and larger hop-count.

However, the difference of the average hop-count between NAVC and AODV is not too large because non-local traffic patterns in which the average distance grows with the network size result in a rapid decrease of per node capacity [27]. By conducting transmit power control, NAVC is able to reduce the interference so that more nodes become available for forwarding packets for others. Consequently, new paths are created and some of them have similar or even shorter length compared to those paths constructed by AODV using the minimum hop-count metric.

\section{Conclusion and Future Work}

In this paper we have presented and evaluated a novel interference aware metric for dense multi-hop wireless networks to improve the overall network performance. This metric can be used at the network layer to facilitate route selection, or the physical layer to conduct transmit power control in a fully distributed manner to extend network lifetime. For both cases, only the interference information from the MAC layer is exploited.

We have shown experimentally that the NAVC-driven AODV is interference aware, where route selection incorporates the effects of the interference in the whole path. It can help to improve the system performance 
in terms of throughput and network lifetime, though using slightly longer paths to deliver packets as compared with the pure AODV.

As a part of our future work, several topics related to NAVC could be further explored: its mapping function to the hop-count when functioning as a metric for routing; its effectiveness when nodes are with high degree of mobility; its ability to handle large-scale ad hoc networks, etc. Besides, we are about to investigate the benefits of interference aware routing under more complex scenarios occurred in sensor networks.

\section{References}

[1] The Network Simulator - ns-2, http://www.isi.edu/nsnam/ns/.

[2] D. Anick, D. Mitra, and M. Sondhi, "Stochastic theory of a data-handling system with multiple sources," The Bell System Technical Journal, vol. 61, no. 8, pp. 1871-1894, Oct 1982.

[3] S. Banerjee and A. Misra, "Minimum energy paths for reliable communication in multi-hop wireless networks," in Proceedings of the 3rd ACM international symposium on Mobile ad hoc networking 8 computing. ACM Press, 2002, pp. 146-156.

[4] C. Bettstetter, "On the Minimum Node Degree and Connectivity of a Wireless Multihop Network," in The ACM Symposium on Mobile Adhoc Networking and Computing (MOBIHOC 2002), Lausanne, Switzerland, June 9-11 2002.

[5] G. Bianchi, "Performance analysis of the ieee 802.11 distributed coordination function," IEEE Journal on selected areas in communications special issue on recent advances in wireless multimedia, vol. 18, no. 3, pp. 535-547, March 2000.

[6] J. Broch, D. A. Maltz, D. B. Johnson, Y.-C. Hu, and J. Jetcheva, "A performance comparison of multi-hop wireless ad hoc network routing protocols," in Proceedings of the 4th annual ACM/IEEE international conference on Mobile computing and networking. ACM Press, 1998, pp. 85-97.

[7] R. Brockett, W. Gong, and Y. Guo, "Stochastic analysis for fluid queueing systems," in IEEE Conference on Decision and Control (CDC) 99, vol. 3, Dec 1999, pp. 3077-3082.

[8] B. Chen, K. Jamieson, H. Balakrishnan, and R. Morris, "Span: An Energy-Efficient Coordination Algorithm for Topology Maintenance in Ad Hoc Wireless Networks," in ACM MobiCom 2001, Rome, Italy, July 16-21 2001, pp. 85-96.

[9] S. R. Das, C. E. Perkins, and E. M. Royer, "Performance Comparison of Two On-demand Routing Protocols for Ad Hoc Networks," in Infocom 2000, Tel-Aviv, Israel, March 2000.

[10] D. S. J. De Couto, D. Aguayo, J. Bicket, and R. Morris, "A high-throughput path metric for multi-hop wireless routing," in Proceedings of the 9th annual international conference on Mobile computing and networking. ACM Press, 2003, pp. 134-146.

[11] D. S. J. De Couto, D. Aguayo, B. A. Chambers, and R. Morris, "Performance of multihop wireless networks: Shortest path is not enough," in Proceedings of the First Workshop on Hot Topics in Networks (HotNets-I). Princeton, New Jersey: ACM SIGCOMM, October 2002.

[12] O. Dousse, F. Baccelli, and P. Thiran, "Impact of interferences on connectivity in ad hoc networks," in Proceedings of Infocom, San Francisco, April 2003.

[13] O. Dousse and P. Thiran, "Connectivity vs capacity in dense ad hoc networks," in Proceedings of IEEE Infocom, Hong Kong, March 2004. 
[14] R. Draves, J. Padhye, and B. Zill, "Comparison of routing metrics for static multi-hop wireless networks," in ACM SIGCOMM' 2004, Portland, OR, August 2004.

[15] L. M. Feeney and M. Nilsson, "Investigating the Energy Consumption of a Wireless Network Interface in an Ad Hoc Networking Environment," in IEEE INFOCOM 2001, vol. 3, Anchorage, Alaska, April 22-26 2001, pp. 1548-1557.

[16] G. Ferrari and O. K. Tonguz, "Minimum number of neighbors for fully connected uniform ad hoc wireless networks," in IEEE International Conference on Communications (ICC 2004), Paris, France, June 2004.

[17] J. Gomez, A. T. Campbell, M. Naghshineh, and C. Bisdikian, "PARO: Supporting Dynamic Power Controlled Routing in Wireless Ad Hoc Networks," to be published in ACM/Kluwer Journal on Wireless Networks (WINET), 2003.

[18] C. M. Group, "CMU monarch extensions to ns," http://www.monarch.cs.cmu.edu/.

[19] P. Gupta and P. R. Kumar, "The capacity of wireless networks," IEEE Transactions on Information Theory, vol. IT-46, no. 2, pp. 388-404, March 2000.

[20] R. Hekmat and P. V. Mieghem, "Interference in wireless multi-hop ad-hoc networks and its effect on network capacity," Wireless Networks, vol. 10, pp. 389-399, 2004.

[21] IEEE, "Wireless lan medium access control (mac) and physical layer (phy) specifications," IEEE Std 802.11-1997, Nov 1997.

[22] Q. Z. W. Z. J. Chen, S.-H. Chan and J. Chen, "Power adaptation for starvation avoidance (pasa): A distributed algorithm to improve short-term fairness for multimedia traffic in mobile ad hoc networks," IEEE Journal on selected areas in communications special issue on recent advances in wireless multimedia, to appear.

[23] K. Jain, J. Padhye, V. N. Padmanabhan, , and L. Qiu, "Impact of interference on multi-hop wireless network performance," in Proc. of the ACM MOBICOM, San Diego, CA, Sept. 2003.

[24] D. B. Johnson, "Routing in ad hoc networks of mobile hosts," in Proceedings of the IEEE Workshop on Mobile Computing Systems and Applications, 1994, pp. 158-163.

[25] D. B. Johnson, D. A. Maltz, Y.-C. Hu, and J. G. Jetcheva, "The dynamic source routing protocol for mobile ad hoc networks (DSR)," http://www.ietf.org/internet-drafts/draft-ietf-manet-dsr-07.txt, February 2002, work in progress. [Online]. Available: http://www.ietf.org/internet-drafts/draft-ietfmanet-dsr-07.txt

[26] V. Kawadia and P. R. Kumar, "Power Control and Clustering in Ad Hoc Networks," in IEEE INFOCOM 2003, vol. 1, San Francisco, CA, March 30-April 3 2003, pp. 459-469.

[27] J. Li, C. Blake, D. S. De Couto, H. I. Lee, and R. Morris, "Capacity of ad hoc wireless networks," in Proceedings of the 7th annual international conference on Mobile computing and networking. ACM Press, 2001, pp. 61-69.

[28] L. Ma, Q. Zhang, Y. Xiong, and W. Zhu, "Interference aware metric for dense multi-hop wireless networks," in IEEE International Conference on Communications (ICC 2005), Seoul, Korea, May 2005.

[29] M. K. Marina and S. R. Das, "Routing Performance in the Presence of Unidirectional Links in Multihop Wireless Networks," in The ACM Symposium on Mobile Adhoc Networking and Computing (MOBIHOC 2002), Lausanne, Switzerland, June 9-11 2002. 
[30] J. Monks, J.-P. Ebert, W.-M. W. Hwu, and A. Wolisz, "Energy saving and capacity improvement potential of power control in multi-hop wireless networks," Comput. Networks, vol. 41, no. 3, pp. 313$330,2003$.

[31] S. Narayanaswamy, V. Kawadia, R. S.Sreenivas, and P. R. Kumar, "Power Control in Ad-Hoc Networks: Theory, Architecture, Algorithm and Implementation of the COMPOW Protocol," in European Wireless 2002, Florence, Italy, Feb 25-28 2002, pp. 156-62.

[32] C. E. Perkins, E. M. Belding-Royer, and S. R. Das, "Ad hoc on-demand distance vector (AODV) routing," http://www.ietf.org/internet-drafts/draft-ietf-manet-aodv-10.txt, January 2002, work in progress. [Online]. Available: http://www.ietf.org/internet-drafts/draft-ietf-manet-aodv-10.txt

[33] T. J. Shepard, "A channel access scheme for large dense packet radio networks," in Conference proceedings on Applications, technologies, architectures, and protocols for computer communications. ACM Press, 1996, pp. 219-230.

[34] S. Singh, M. Woo, and C. S. Raghavendra, "Power-aware routing in mobile ad hoc networks," in Proceedings of the 4 th annual ACM/IEEE international conference on Mobile computing and networking. ACM Press, 1998, pp. 181-190.

[35] F. Xue and P. Kumar, "The number of neighbors needed for connectivity of wireless networks," Wireless Networks, vol. 10, no. 2, pp. 169-181, March 2004.

[36] Q. Zhang, C. Guo, Z. Guo, and W. Zhu, "Efficient mobility management for vertical handoff between wwan and wlan," IEEE Communications Magazine, vol. 41, pp. 102-108, Nov 2003. 